\title{
Reduction in Sidelobe Level in Ultracompact Arrayed Waveguide Grating Demultiplexer Based on Si Wire Waveguide
}

\author{
Fumiaki OHNO, Kosuke SASAKI, Ayumu MOTEGI and Toshihiko BABA \\ Department of Electrical and Computer Engineering, Yokohama National University, 79-5 Tokiwadai, \\ Hodogaya-ku, Yokohama 240-8501, Japan
}

We designed and fabricated $70 \times 75 \mu \mathrm{m}^{2}$ arrayed waveguide grating demultiplexer consisting of $\mathrm{Si}$ slab and wire waveguides on a silicon-on-insulator substrate. By optimizing the connection between components and the layout of arrayed waveguides, internal light scattering and the increase in phase error were suppressed. As a result, clear demultiplexing characteristics were observed with a channel spacing of $8 \mathrm{~nm}$ and a sidelobe level of $-22 \mathrm{~dB}$ in the wavelength range from 1.5 to $1.6 \mu \mathrm{m}$.

Keywords: Si photonics, photonic wire, high-index contrast, photonic integration, arrayed waveguide grating, AWG

\section{Introduction}

A Si photonic wire waveguide (PWW) has an ultrahigh relative refractive index difference $\Delta$ of more than $40 \%$ between its Si core and low index claddings, and so realizes micron-radius bend and various micro-optic components. ${ }^{1-7)}$ Therefore, it is effective for use as dense optical wiring in integrated optic circuits, and in recent years, its applications to compact multi/demultiplexers have been studied. ${ }^{8-13)}$

Now, arrayed waveguide gratings (AWGs) based on silica waveguides are widely used as high performance multi/demultiplexers for wavelength division multiplexing systems. ${ }^{14)}$ However, their size is on the $\mathrm{cm}^{2}$ order owing to their low $\Delta$ of maximally $5 \%$ and the $\mathrm{mm}-\mathrm{cm}$-radius bends of silica waveguides. InP-based semiconductor high mesa waveguides enable the formation of $\mathrm{mm}^{2}$-sized AWGs owing to their ultrahigh- $\Delta$ sidewalls. ${ }^{15)}$ Si PWW AWGs are smaller, that is, on the $100 \times 100$ $\mu \mathrm{m}^{2}$ order, owing to its ultrahigh $\Delta$ at all boundaries. Previously, we reported the first fabrication of a $110 \times 90 \mu^{2}$ Si PWW AWG on a silicon-on-insulator (SOI) substrate and demonstrated its preliminary demultiplexing characteristics. ${ }^{11)}$ However, the sidelobe level of each demultiplexed spectrum was greater than $-5 \mathrm{~dB}$ even in the best spectrum and the excess loss of the AWG excluding the coupling loss with an external optical setup was greater than $12 \mathrm{~dB}$. These results were primarily caused by unwanted scattering and resonance inside the device. Recently, for the AWG, we further reduced its size to $70 \times 60 \mu \mathrm{m}^{2}$ and improved its performance by reducing scattering and resonance. ${ }^{16)}$ However, its sidelobe level was still limited to between $-13 \mathrm{~dB}$ and $-8 \mathrm{~dB}$. In this study, we particularly focused on the sidelobe issue and optimized the AWG's design.

In this paper, we first present the total analysis of light propagation in the Si PWW AWG by a finite-difference time-domain (FDTD) method in $\S 2$. Then in $\S 3$, we show an improved design after discussing three issues, i.e., the generation of an ideal Gaussian beam at the connecting portion between the input waveguide and the first slab waveguide, a reduction in scattering loss at connecting portions between slab and arrayed waveguides, and a reduction in phase error in actual arrayed waveguides with small disordering. In $\S 4$, we describe the fabrication and evaluation of the newly designed device, and finally show that the sidelobe level was reduced to $-22 \mathrm{~dB}$ in the best spectrum.

\section{Total FDTD Analysis}

The Si core of the PWW used in our study was $0.32 \mu \mathrm{m}$ thick and $\sim 0.45 \mu \mathrm{m}$ wide, which satisfy the single-mode condition at a wavelength $\lambda$ of $1.55 \mu \mathrm{m}$. In addition to this, the following structural details were employed in our previous study of the PWW AWG. ${ }^{16)}$ Slab waveguides for light distribution and focusing were laterally opened to the outer region so that the internal reflection was suppressed; their focal length $f$ was $25 \mu \mathrm{m}$. Arrayed waveguides consisted of smoothly curved PWWs whose pitch $p$ and length difference $\Delta L$ between adjacent PWWs were 1.0 and $6.06 \mu \mathrm{m}$, respectively. These conditions correspond to a diffraction order $m$ of 10 and a free spectral range (FSR) of $\sim 87 \mathrm{~nm}$. By setting the pitch of output waveguides $P$ to $1.5 \mu \mathrm{m}$, the channel spacing $\Delta \lambda$ was expected to be 11 
$\mathrm{nm}$.

In the FDTD analysis, we fully modeled the above device using two-dimensional (2D) device patterns with equivalent indexes of 3.06 for the Si region and 1.0 for the surrounding air region. To save the computer memory space and computation time and satisfy the finite difference condition at $\lambda$ $\sim 1.55 \mu \mathrm{m}$, the Yee cell size was set to $40 \times 40 \mathrm{~nm}^{2}$. Therefore, the total number of cells was 7.7 million. On this condition, the waveguide width of $0.44 \mu \mathrm{m}$ should be digitized using 11 cells. However, a digital error of maximally one cell is introduced into bends and angled patterns. Figure 1(a) shows the demultiplexing operation of the AWG. Here, in-plane polarized (TE-polarized) continuous wave light at $\lambda=1.55 \mu \mathrm{m}$ is launched into the input waveguide. The input light is expanded in slab waveguide A, distributed to arrayed waveguides, focused in slab waveguide B and coupled to output waveguides. The FSR was estimated to be $100 \mathrm{~nm}$, which agrees well with the designed value. In this figure, however, the expanded beam in slab waveguide A exhibits a deformed pattern. Also, unwanted scattering and diffraction are observed at connecting portions between the slab and arrayed waveguides. Because the slab waveguides are laterally opened, the scattered light efficiently escapes from the device, and so its direct coupling into output waveguides is small. However, it cannot be ignored, when the target sidelobe level is lower than $-20 \mathrm{~dB}$. Also, the unwanted diffraction deforms the focused light spot and causes a high sidelobe level. In this calculation, the sidelobe levels totally determined by these effects range from -8 to $-15 \mathrm{~dB}$, as shown in Fig. 1(a).

Figure 1(b) shows calculation results for the new design in this study. The same demultiplexing function is observed, and sidelobe level is improved from $-16 \mathrm{~dB}$ to $-20 \mathrm{~dB}$. The process of obtaining this design is explained in the following sections.

\section{Issues and New Design}

First, we discuss three issues one by one, and then show the details of the new design.

\subsection{Gaussian beam generation}

In the input waveguide, guided light is strongly confined within a spot width of less than $0.5 \mu \mathrm{m}$ at $\lambda \sim 1.55 \mu \mathrm{m}$. If the waveguide is directly connected to slab waveguide $\mathrm{A}$, the light beam in the slab is expanded too widely. Besides this, we were anxious about whether the connecting portion having abrupt changes of ultrahigh $\Delta$ boundaries would cause pronounced light scattering and a deformation of the beam profile. An ideal beam profile in slab waveguide A, which suppresses the sidelobe in the AWG, is a Gaussian profile, although some other profiles are known to be effective for producing a flat top and sharp roll-off spectrum. To generate an appropriate Gaussian beam, we inserted a parabolic taper, which expands the guided light beam without exciting higher-order modes. ${ }^{17)}$ For the precise simulation of transient light propagation, we used the three-dimensional (3D) FDTD method. We assumed refractive indexes of the $\mathrm{Si}$ core, $\mathrm{SiO}_{2}$ lower cladding, and air side and upper claddings to be 3.46, 1.44, and 1.0, respectively, and the TE-polarized input light to be at $\lambda=1.55 \mu \mathrm{m}$. Figure 2 shows the modal profile in the slab waveguide, provided that the taper length $l_{1}$ is $3 \mu \mathrm{m}$ and the taper width $w_{1}$ is taken as a parameter. Here, the profile is observed on an arc showing the best fit to the wavefront of the expanded beam. The best Gaussian-like beam is observed for $w_{1}=1.48 \mu \mathrm{m}$, as shown in Fig. 2. The full divergence angle $2 \theta_{\mathrm{b}}$ at the $1 / e^{2}$ intensity of the beam is almost inversely proportional to $w_{1}$ and is $\sim 40^{\circ}$ for $w_{1}=1.48 \mu \mathrm{m}$. Figure 2 also shows the spatial Fourier transform of the beam profile, which gives a rough estimate of the demultiplexed spectrum in the AWG. Here, the angle detectable by arrayed waveguides $2 \theta_{\mathrm{a}}$ is taken as a parameter. The sidelobe level is insensitive to $2 \theta_{\mathrm{a}}$, once $2 \theta_{\mathrm{a}}>2 \theta_{\mathrm{b}}$ is satisfied. A low sidelobe level of $-31 \mathrm{~dB}$ was estimated for the optimum $w_{1}$. Therefore, the light scattering at the connecting portion will not be crucial if other issues discussed below have a higher sidelobe level.

\subsection{Reduction in scattering loss}

The reduction in scattering loss at connecting portions between the slab and arrayed waveguides is an important issue for any AWGs. Because direct coupling causes a significant loss, various connection tapers have been discussed. ${ }^{14,18)}$ In this study, we employed a parabolic taper and optimized 
the structure by the 3D FDTD method. To obtain better calculation convergence, we reduced the Yee cell size to $20 \times 20 \times 20 \mathrm{~nm}^{3}$. To save computation time, we used a simplified rectangular model, as shown in the inset of Fig. 3. It has a width equal to the pitch $p$ of arrayed waveguides, and includes only the slab waveguide and one PWW with the taper. By applying a periodic boundary condition to the sidewalls of the model, the connection between the infinitely wide slab and the infinite number of arrayed waveguides can be calculated. Here, the curved boundary in the AWG is approximated by the straight boundary. The error should be negligible owing to curvature radius being much larger than the waveguide width. The excitation was uniformly given for the TE polarization in the slab, and relative light intensity in the arrayed waveguide was evaluated. First, we noticed that the scattering loss is not sensitive to the taper length $l_{2}$ and the pitch $p$. Then, we fixed $l_{2}=3 \mu \mathrm{m}$ and $p=1 \mu \mathrm{m}$, and calculated the dependence on the taper width $w_{2}$, as shown in Fig. 3. Light transmittance is improved as $w_{2}$ approaches $p$ (in other words, the gap between tapers is reduced). The minimum loss is estimated to be $0.8 \mathrm{~dB}$, when $w_{2}$ is equal to $p$ (zero gap).

\subsection{Reduction in phase error}

When the arrayed waveguides fluctuate in width, the phase front in them is disordered. This leads to the deformation of the focused spot and the enhancement in sidelobe level. For the quantitative evaluation of this effect, we first simply estimated the demultiplexed spectrum by considering a Gaussian beam profile with $2 \theta_{b}=\sim 40^{\circ}$ (light in slab waveguide A), digitizing it with a minimum angular unit $\Delta \theta=1.5^{\circ}$ (distribution of light into arrayed waveguides), adding random phase shifts within a specified maximum for each digitized unit (phase error in arrayed waveguides) and the Fourier transformation (focusing in slab waveguide B). As a result, sidelobe levels of $-22,-17,-14$, and $-8 \mathrm{~dB}$ were expected when maximum phase error was set to $30,60,90$, and $150^{\circ}$, respectively.

Next, we calculated the phase shift in each arrayed waveguide in the total 2D FDTD simulation of the AWG. One difference from the calculation in Section II is that an ideal Gaussian beam was directly incident to the arrayed waveguides so that the influence of the beam profile was eliminated. As noted previously, a digital error of maximally one Yee cell $(=40 \mathrm{~nm})$ is automatically introduced into the width of curved arrayed waveguides in the calculation model. This digital error does not precisely simulate the small nonuniformity expected in fabricated waveguides. However, this simulation enables us to qualitatively evaluate phase error and its influence to the spectral characteristics. Figure 4(a) shows the field distribution of guided light near the end of arrayed waveguides, where the diffraction order $m$ and increment $\Delta L$ are set to 10 and $6.06 \mu \mathrm{m}$, respectively, as assumed in Fig. 1(a). All antinodes in the guided mode in the arrayed waveguides seem to be on an ideal isophase curve (white dashed curve), which is given by a circle centered at the focal point of slab waveguide B. However, a careful evaluation of antinodal positions revealed that the phase front is slightly deformed. The phase error measured from the iso-phase curve is shown by the dashed line in Fig. 4(b). In this evaluation, the phase resolution is limited to $30^{\circ}$ by the ratio of the Yee cell size to the mode size. For $m=10$, maximum errors from $-60^{\circ}$ to $+90^{\circ}$ are observed with a background level of $\pm 30^{\circ}$. Therefore, sidelobe levels from -14 to $-17 \mathrm{~dB}$ are expected from the above discussion. It roughly corresponds to the calculated values from -8 to $-15 \mathrm{~dB}$ in the total FDTD calculation shown in Fig. 1(a).

The simplest way to reduce the absolute value of phase error is to shorten arrayed waveguides. In the design shown in Fig. 1(a), however, not all the arrayed waveguides can be equally shortened, because the innermost waveguide is already very short $(=6 \mu \mathrm{m})$. Therefore, we reduced $m$ so that the outer waveguides are more shortened than the inner waveguides. We finally designed the AWG in Fig. 1(b) with $m=5$ and $\Delta L=3.03 \mu \mathrm{m}$. Here, slab waveguide B is angled by $60^{\circ}$ against slab waveguide A to realize the different shortening lengths of arrayed waveguides, maintaining a minimum $p$ of $0.9 \mu \mathrm{m}$. The phase error calculated for this design is shown by the solid line in Fig. 4(b). Maximum error is reduced to $\pm 30^{\circ}$, and a sidelobe level of $-22 \mathrm{~dB}$ is expected, which roughly corresponds to the calculated value from -16 to $-20 \mathrm{~dB}$ in Fig. 1(b)

\subsection{New design}

In the new design shown in Fig. 1(b), an optimized taper with $l_{1}=3 \mu \mathrm{m}$ and $w_{1}=1.48 \mu \mathrm{m}$ was inserted between the input waveguide and slab waveguide A. The same taper is inserted between 
output waveguides and slab waveguide B so that the device structure and light propagation become symmetric. The focal length $f$ of the slab waveguide is extended to $35 \mu \mathrm{m}$ to compensate for the wavelength resolution reduced by the reduced $m$. The number of arrayed waveguides is 28 , and the detectable beam angle $2 \theta_{\mathrm{a}}$ is $40^{\circ}$. This angle is exactly the Gaussian beam angle $2 \theta_{\mathrm{b}}=40^{\circ}$ in slab waveguide A. The optimum tapers are also inserted between slab and arrayed waveguides with $l_{2}=3$ $\mu \mathrm{m}$ and $w_{2}=0.9 \mu \mathrm{m}$. To reduce phase error, $m=5$ and $\Delta L=3.03 \mu \mathrm{m}$ are employed, and two slab waveguides are angled by $60^{\circ}$. For these parameters, the expected channel spacing $\Delta \lambda$ is $11 \mathrm{~nm}$, which is the same as that in Fig. 1(a), and the FSR is expanded by a smaller $m$ to $155 \mathrm{~nm}$. As noted above, this design allows a lower sidelobe level of $-20 \mathrm{~dB}$ in the total calculation.

\section{Experiment}

The device shown in Fig. 1(b) was fabricated on a SOI wafer with a $0.32-\mu \mathrm{m}$-thick Si layer by the same process as that in ref. 16). The device was formed by $e$-beam lithography, pattern transfer to a $\mathrm{Cr}$ mask and $\mathrm{CF}_{4}$ inductively coupled plasma etching. In this method, the sidewall roughnesses evaluated by high-resolution scanning electron microscope are typically $10-30 \mathrm{~nm}$. Just for the vertical etching of a thin Si slab, an $e$-beam resist can be directly used as a mask. We tested such a simpler process and confirmed that it reduces sidewall roughness and waveguide loss. However, it was difficult to form fine tapers between slab and arrayed waveguides with a narrow gap by the resist mask process; thus, pronounced scattering loss occurred. In the metal mask process used, a narrow gap of less than $50 \mathrm{~nm}$ was realized and the scattering loss was well suppressed.

In the measurement, tunable laser light was coupled to the input waveguide by two objective lenses. To reduce the facet reflection and internal Fabry-Perot resonance, a taper of $15 \mu \mathrm{m}$ length and $0.2 \mu \mathrm{m}$ tip width was placed at the input end. The light output was observed by an infrared InGaAs camera and measured by an optical power meter. The propagation loss of the linear waveguide was 14 $\mathrm{dB} / \mathrm{mm}$ owing to the above-mentioned roughness at etched sidewalls. However, it is still not the dominant factor in the total loss of the very compact AWG. Figure 5 shows near-field patterns at the ends of output waveguides for different $\lambda$ 's. The output spot clearly shifted with $\lambda$. The FSR was measured to be $140 \mathrm{~nm}$, which is close to the theoretical value. Figure 6 shows the transmission spectra measured for all output waveguides. A fine oscillation of $\sim 2 \mathrm{~dB}$ at the top of each spectrum was caused by the Fabry-Perot resonance. It is negligible if the tip width of the input taper is sufficiently small or a spot size converter is integrated. ${ }^{4)}$ Clear Gaussian spectra were observed with channel spacings $\Delta \lambda$ of $8-10 \mathrm{~nm}$, which is slightly smaller than the designed value. Note that $\Delta \lambda$ is given by $n_{s} p P \lambda / n_{\mathrm{g}} f \Delta L$ in AWGs, where $n_{\mathrm{s}}$ and $n_{\mathrm{g}}$ are the equivalent index of the slab waveguide and the group index of each arrayed waveguide, respectively. Four parameters $p, P, f$, and $\Delta L$, are fixed by the pattern layout, and $n_{\mathrm{s}}$ is determined by the thickness of the top Si layer of the SOI wafer. Therefore, $\Delta \lambda$ might be changed by $n_{\mathrm{g}}$, which is particularly sensitive to the waveguide width in the PWW. ${ }^{1)}$ Because of the reduced $\Delta \lambda$, the spectra of adjacent channels approached each other, and the adjacent channel crosstalk was limited to $-13 \mathrm{~dB}$. However, the sidelobe level of each spectrum was greatly improved from that of the previous spectrum; it was -22 and $-12 \mathrm{~dB}$ in the best and worst spectra. By appropriately controlling $\Delta \lambda$, channel crosstalk can be the same level as the sidelobe level. The ordinate of the graph in Fig. 6 represents the relative output intensity, when the intensity from a $100-\mu \mathrm{m}$-long PWW is used as a reference. The peak values range from -2.5 to $-5.0 \mathrm{~dB}$ among output ports. Insertion loss is mainly due to the loss of the input and output waveguides of nearly $150 \mu \mathrm{m}$ total length. By neglecting propagation loss, the loss of the AWG was estimated to be less than $2 \mathrm{~dB}$ for the best spectrum.

\section{Conclusions}

We designed and fabricated a $70 \times 75 \mu^{2}$ Si PWW AWG demultiplexer on a SOI wafer. We particularly aimed to reduce the sidelobe level, and investigated the generation of appropriate Gaussian beams, the reduction in the connection loss of slab and arrayed waveguides by inserting tapers, and the reduction in phase error by reducing diffraction order and shortening arrayed waveguides. In the fabricated device, we observed a channel spacing of $8 \mathrm{~nm}$, an FSR of $140 \mathrm{~nm}$, a 
sidelobe level of $-22 \mathrm{~dB}$ and a device loss of less than $2 \mathrm{~dB}$ in the best spectrum. If the etching process is improved so that smooth waveguide sidewalls and fine tapers are simultaneously realized, phase error will be further reduced and sidelobe level will reach an almost practical level of $-30 \mathrm{~dB}$, which is determined from the initial Gaussian beam profile.

\section{Acknowledgments}

This work was supported by the IT Program and the 21st Century COE Program of the Ministry of Education, Culture, Sports, Science and Technology, and a Grant-In-Aid from the Japan Society for the Promotion of Science.

\section{References}

1) A. Sakai, G. Hara and T. Baba: Jpn. J. Appl. Phys. 40 (2001) L383-L385.

2) K. K. Lee, D. R. Lim, L. C. Kimmerling, J. Shin and F. Cerrina: Opt. Lett. 26 (2001) 1888.

3) A. Sakai, T. Fukazawa and T. Baba, IEICE Trans. Electron. E85-C (2002) 1033.

4) T. Shoji, T. Tsuchizawa, T. Watanabe, K. Yamada and H. Morita: Electron. Lett. 38 (2002) 1669.

5) T. Fukazawa, T. Hirano, F. Ohno and T. Baba: Jpn. J. Appl. Phys. 43 (2004) 646.

6) Y. A. Vlasov and S. J. McNab: Opt. Express 12 (2004) 1622.

7) W. Bogaerts, R. Baets, P. Dumon, V. Wiaux, S. Beckx, D. Taillaert, B. Luyssaert, J. Van Campenhout, P. Bienstman and D. Van Thourhout: J. Lightwave Technol. 23 (2005) 401.

8) J. Foresi, P. Villeneuve, J. Ferrara, E. Thoen, G. Steinmeyer, S. Fan, J. Joannopoulos, L. Kimerling, H. Smith and E. Ippen: Nature 390 (1997) 143.

9) B. E. Little, J. S. Foresi, G. Steinmeyer, E. R. Thoen, S. T. Chu, H. A. Haus, E. P. Ippen, L. C. Kimeling and W. Greene: IEEE Photonics Technol. Lett. 10 (1998) 549.

10) K. Yamada, T. Shoji, T. Tsuchizawa, T. Watanabe, J. Takahashi and S. Itabashi: Opt. Lett. 28 (2003) 1663.

11) T. Fukazawa, F. Ohno and T. Baba: Jpn. J. Appl. Phys. 43 (2004) L673.

12) V. R. Almeida, C. A. Barrios, R. R. Panepucci and M. Lipson: Nature 43 (2004) 1081.

13) F. Ohno, T. Fukazawa and T. Baba: Jpn. J. Appl. Phys. 44 (2005) 5322.

14) K. Okamoto: Fundamentals of Optical Waveguides (Academic Press, New York, 2000).

15) M. Kohtoku, H. Sanjoh, S. Oku, Y. Kadota, Y. Yoshikuni and Y. Shibata: Electron. Lett. 33 (1997) 1786.

16) K. Sasaki, F. Ohno and T. Baba: Electron. Lett. 41 (2005) 801.

17) W. K. Burns, A. F. Milton and A. B. Lee: Appl. Phys. Lett. 30 (1977) 28.

18) J. H. den Besten, M. P. Dessens, C. G.P. Herben, X. J. M. Leijtens, F. H. Groen, M. R. Leys and M. K. Smit: IEEE Photonics Technol. Lett. 14 (2002) 62. 


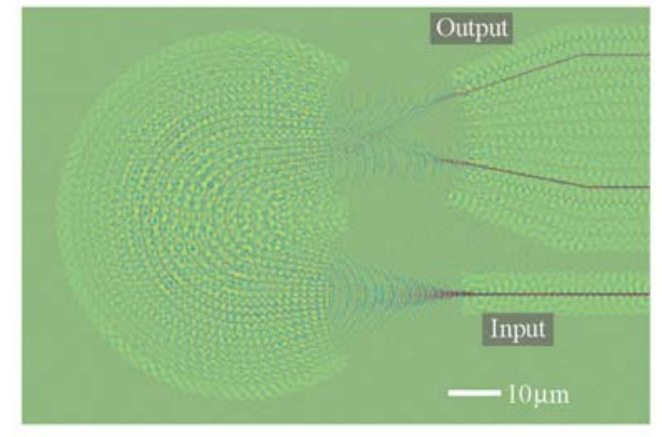

(a)
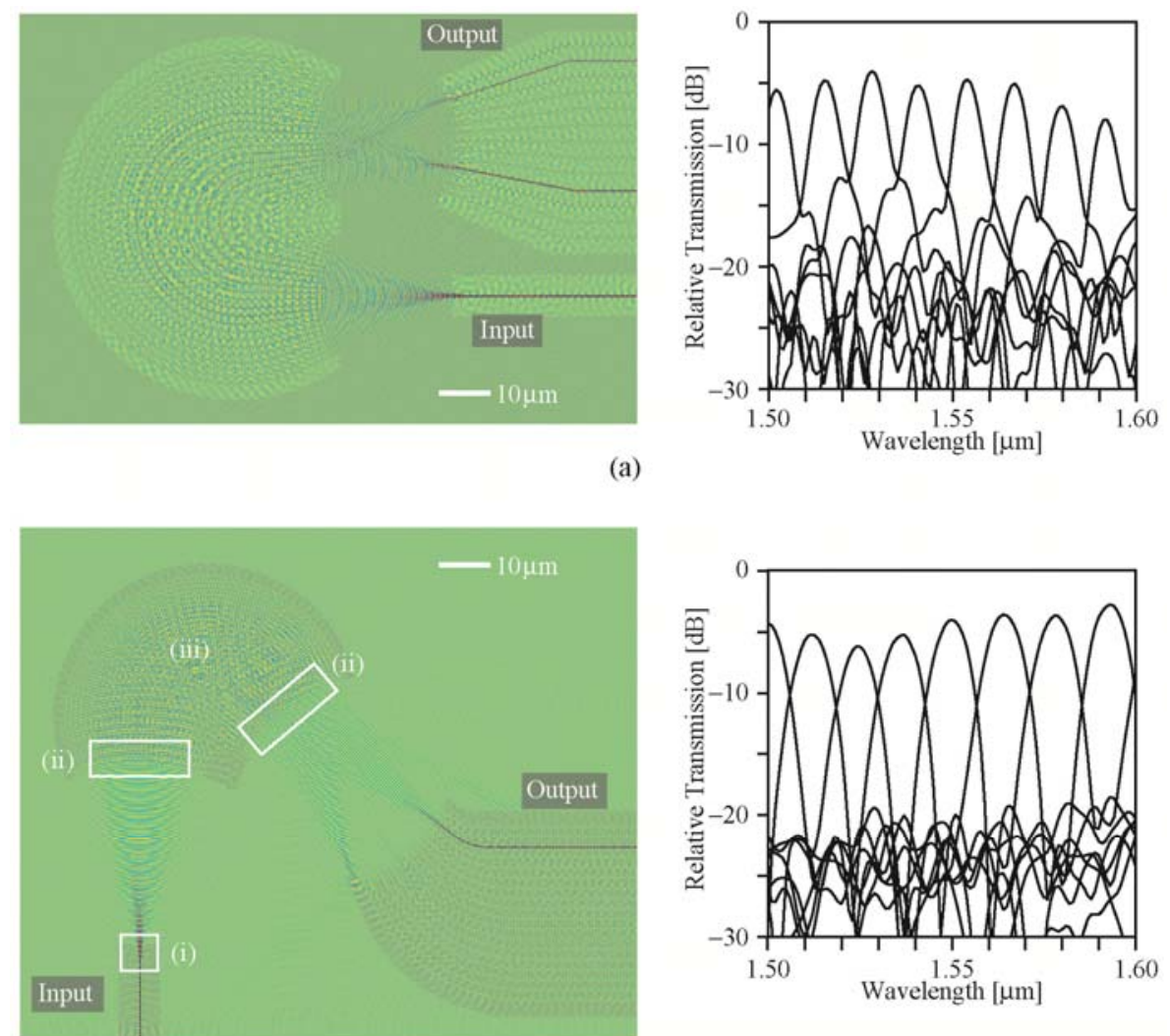

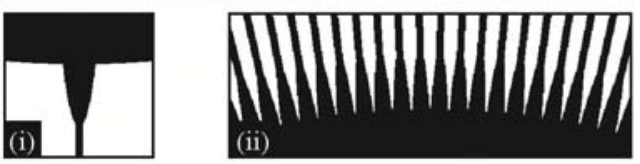

(b)

Fig. 1. Field distribution of light in AWGs and transmission spectra calculated by 2D FDTD method. (a) Previous design with $m=10$. (b) New design with $m=5$.

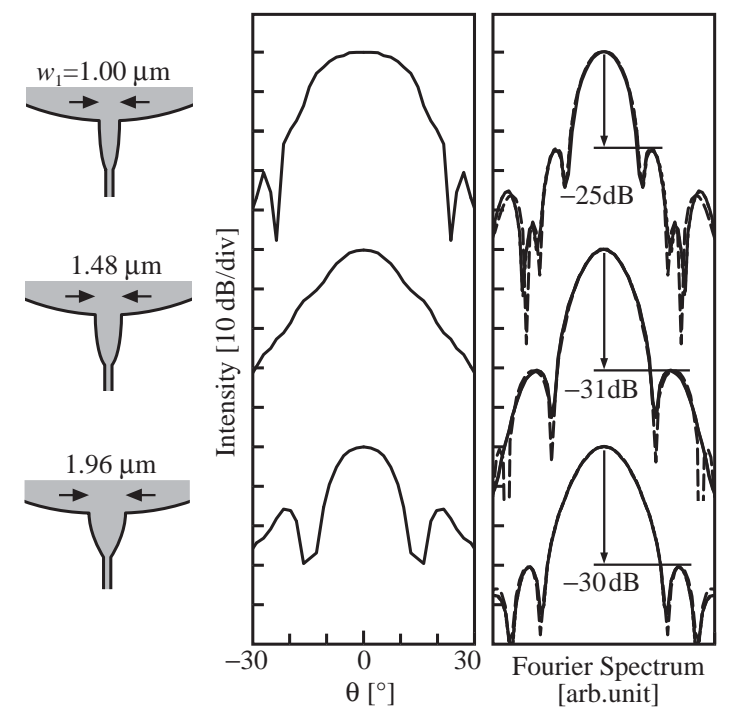

Fig. 2. Field profile of light beam in slab waveguide A (left) and corresponding Fourier spectrum (right) when parabolic taper is inserted between the input waveguide and slab waveguide A. Three different taper widths $w_{1}$ are considered. The Fourier spectra were calculated for three different detectable angles $2 \theta_{\mathrm{a}}$ of arrayed waveguides $\left(180,90\right.$, and $\left.40^{\circ}\right)$, which are shown by solid, dashed and dotted curves, respectively. The dotted curves almost overlap with the other curves. 


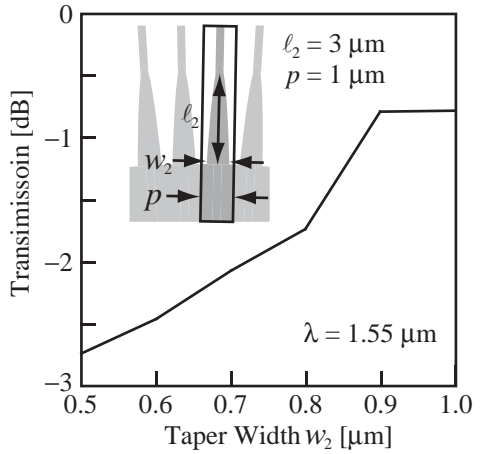

Fig. 3. Transmission efficiency with width $w_{2}$ of parabolic taper inserted between slab and arrayed waveguides calculated by 3D FDTD method. The thick line in the inset shows the unit cell calculation model.

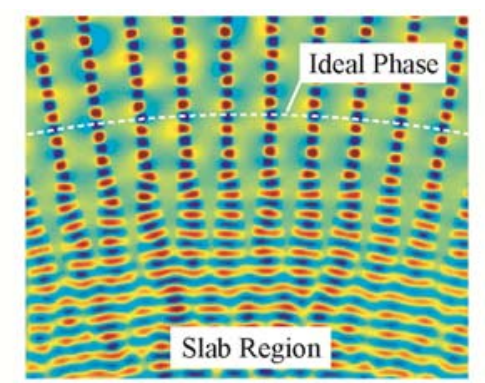

(a)

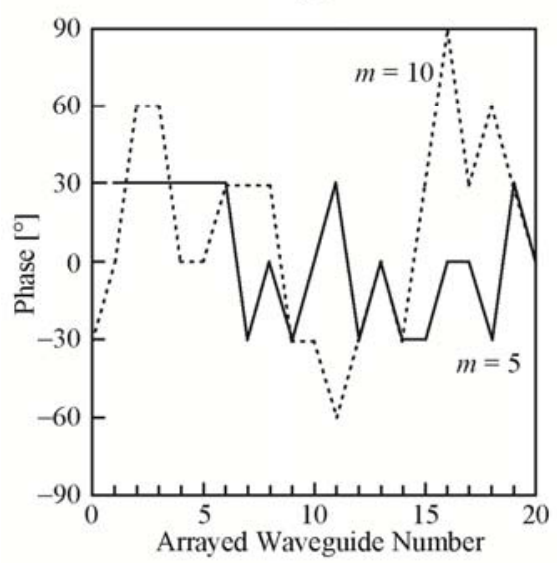

(b)

Fig. 4. Phase of light near end of arrayed waveguides. (a) Field distribution. (b) Phase measured from ideal iso-phase curve in arrayed waveguides. The dashed and solid lines denote devices with $m=10$ and 5, respectively.
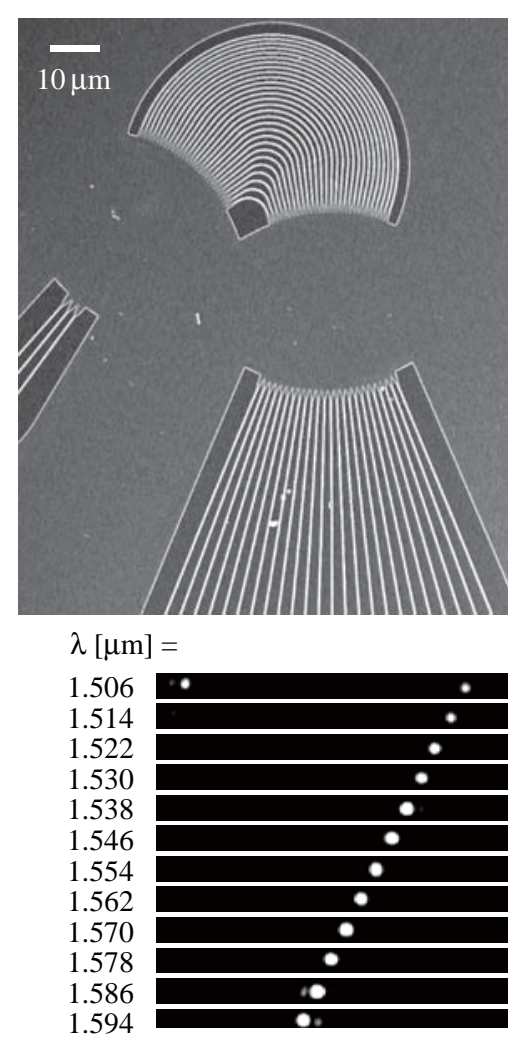

Fig. 5. Scanning electron micrograph of fabricated AWG and near-field patterns of output light for different $\lambda$ 's.

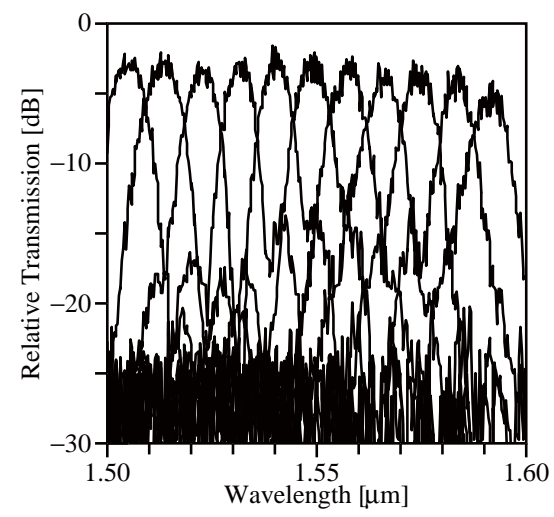

Fig. 6. Measured transmission spectra for 11 output ports. 